\title{
Pseudolayered structure of the columnar B1 phase of bent-core liquid crystals
}

\author{
J. Martinez-Perdiguero, ${ }^{1}$ J. Etxebarria, ${ }^{2}$ C. L. Folcia, ${ }^{2}$ J. Ortega, ${ }^{3}$ N. Gimeno, ${ }^{4}$ and M. B. Ros ${ }^{4}$ \\ ${ }^{1}$ Departamento de Micro- y Nanotecnología, Tekniker, Eibar 20600, Spain \\ ${ }^{2}$ Departamento de Física de la Materia Condensada, Facultad de Ciencia y Tecnología, Universidad del País Vasco, Apdo. 644, \\ 48080 Bilbao, Spain \\ ${ }^{3}$ Departamento de Física Aplicada II, Facultad de Ciencia y Tecnología, Universidad del País Vasco, Apdo. 644, 48080 Bilbao, Spain \\ ${ }^{4}$ Instituto de Ciencia de Materiales de Aragón, Química Orgánica, Facultad de Ciencias, Universidad de Zaragoza-CSIC, \\ 50009 Zaragoza, Spain
}

(Received 12 April 2010; revised manuscript received 30 June 2010; published 18 October 2010)

\begin{abstract}
Three bent-core liquid-crystal compounds presenting the unusual columnar B1 to tilted polar smectic (Sm-CP or B2) phase transition are studied through small-angle $\mathrm{x}$-ray diffraction on aligned samples. The well-established character of the smectic phase helps to characterize the structural changes during the transition. A mechanism for this transition is proposed, which accounts for the low enthalpy usually observed in this process. The B1 phase is found to possess a great degree of lamellarization, with pseudosmectic ferroelectric layers along the crystallographic (11) planes. As a consequence of these observations a distribution of the block polarizations in the columnar structure is proposed.
\end{abstract}

DOI: 10.1103/PhysRevE.82.041706

PACS number(s): 61.30.-v, 61.05.cf, 64.70.mj

\section{INTRODUCTION}

For more than a decade now, bent-core liquid crystals have attracted great attention due to their unusual polar and chiral properties stemming from their peculiar shapes. The hindered rotation around the long molecular axis gives rise to new nonclassical mesophases exclusive of these materials. Among the different orderings, there exist smectic polar phases and, depending on the degree of frustration in the layer formation, undulated smectic and columnar phases [1-3]. In addition, all of them present in their turn different possibilities of polar order arrangement that increases greatly the structural richness. The bent-core mesophases have been designated as B1-B8, but it has been seen that some of these names actually group different structures and, in other cases, different names denote similar structures. Therefore, this nomenclature results unsuitable and, consequently, will be probably abandoned in the future.

The tilted polar smectic ( $\mathrm{Sm}-\mathrm{CP}$ or B2) phases have been widely studied, and their structures are well established. It has been reported that when polarization splay is present, it forces the appearance of defects. To accommodate these defects the layers either undulate (undulated smectic or B7 phases) or break completely to form columns (columnar B1 phases) $[4,5]$. How many different structures are under these names (B1 and B7) and their characteristics are still subject of research.

In this respect, great progress has been achieved in the study of the columnar phases, and the shape and arrangement of the constituent blocks have been determined in many cases by $\mathrm{x}$-ray diffraction studies. However, the arrangement of the polarization direction of the blocks is not accessible by standard $\mathrm{x}$-ray techniques due to the liquid disorder inside the columns, and therefore there are several structures compatible with a given charge-density map (see some basic structural models for the B1 phase in Fig. 1; further molecular organizations are described in Refs. [2,6,7]). Unambiguous information about the block polarization can only be extracted by resonant x-ray diffraction experiments [8] and, up to now, no results have been published in this respect. However, the study of the B1-B2 transition by using X-ray standard techniques can shed some light onto some characteristics of the B1 structure on the basis of the better-known features of the B2 phase.

A direct columnar to $\mathrm{Sm}-\mathrm{CP}$ transition is rather unusual and very few compounds have been reported to posses such dimorphism [9-13]. In some cases it has been found that a field can also induce this sequence $[6,9,12,13]$. A priori, this transition from columnar to smectic phase would suppose a huge structural change but, surprisingly, the transition enthalpy observed by differential scanning calorimetry is very

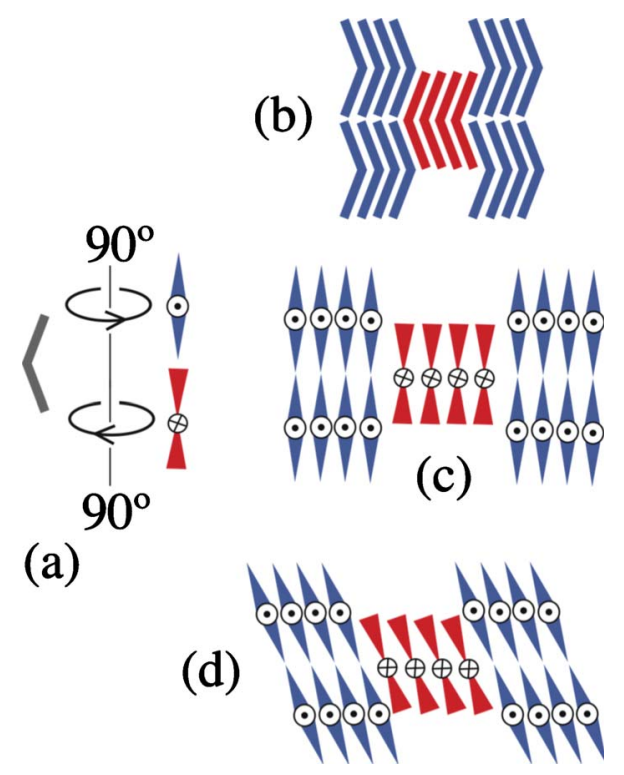

FIG. 1. (Color online) (a) Schematic representation of bent-core molecules and basic structural models proposed for the B1 phase. (b) represents the traditional $\mathrm{B} 1$ phase, (c) is the so-called $\mathrm{B} 1$ reversed $\left(\mathrm{B} 1_{\text {rev }}\right)$ or columnar rectangular $\left(\mathrm{Col}_{\mathrm{r}}\right)$ phase, and $(\mathrm{d})$ is the tilted oblique $\mathrm{B} 1$ reversed phase, $\mathrm{B} 1_{\text {revtilt }}$, or $\mathrm{Col}_{\mathrm{ob}}$. 
small $\left(\sim 0.5 \mathrm{~kJ} \mathrm{~mol}^{-1}[14]\right)$, meaning that the change cannot be too drastic.

In this paper, three compounds presenting the B1-B2 dimorphism were studied by means of $\mathrm{x}$-ray diffraction on aligned samples. The changes in the x-ray diagram at the B1-B2 transition have allowed for a better understanding of the columnar phases, and a model will be proposed for the dipole ordering among the different blocks in these structures. This model is able to give account of the small enthalpy of the B1-B2 transition.

\section{EXPERIMENTAL TECHNIQUES}

Small-angle x-ray scattering measurements were performed in a Bruker Nanostar system equipped with a twodimensional (2D) detector using $\mathrm{Cu} K \alpha$ radiation. Aligned samples were obtained depositing drops of material on mica substrates. The quality of the alignment depended strongly on the material, cooling rate, drop size, and proximity to the surface of the $\mathrm{x}$-ray spot (approximately $100 \mu \mathrm{m}^{2}$ ). It is noteworthy to mention that due to the nature of the drop, there is a symmetry revolution axis perpendicular to the surface, which derives in an artificial vertical twofold axis in the $\mathrm{x}$-ray diagrams.

\section{RESULTS}

The molecular structure together with the phase sequences on cooling of compounds $\mathrm{A}-\mathrm{C}$ is shown in Fig. 2. Structural characterization of compound A can be found in [15]. Field-induced transitions from columnar to smectic were found in compounds B and C (see Refs. [6,9], respectively). Also a structural study of the columnar to smectic transition in compound $\mathrm{C}$ based on $\mathrm{x}$-ray measurements and texture analysis is found in $[14,16]$. In addition, the antiferroelectric character of the three compounds in the columnar and smectic phases has been unambiguously stated in those works.

Figure 3(a) shows the azimuthally integrated 2D x-ray diffraction diagram of compound A vs temperature on cooling, presenting a clear transition from an orthogonal columnar phase (parameters of the rectangular centered cell at $150{ }^{\circ} \mathrm{C}$ are $a=100.9 \AA$ and $c=56.8 \AA$ ) to a smectic structure (with $49.2 \AA$ layer spacing). Figure 3(b) shows a powder-diffraction diagram in a logarithmic scale at $149^{\circ} \mathrm{C}$ within the B1 phase. As can be seen the reflections indexed as (11) and (22) at the columnar phase are preserved in the transition to the smectic structure $[(01)$ and (02) peaks], while the rest of the peaks disappear. Consequently, the transition takes place in such a way that the layer spacing of the smectic phase originates continuously from the (11) spacing of the columnar structure. Moreover, the nonintegrated 2D diagrams in Figs. 3(c) and 3(d) show that the alignment is kept during the transition. Figure 4 shows the procedure followed for the peak indexation. As mentioned above, there is an artificial twofold axis in the 2D diagrams because in all cases two sample domains related by a vertical twofold axis participate in the generation of the observed x-ray pattern. These two domains appear as a consequence of the revolu-

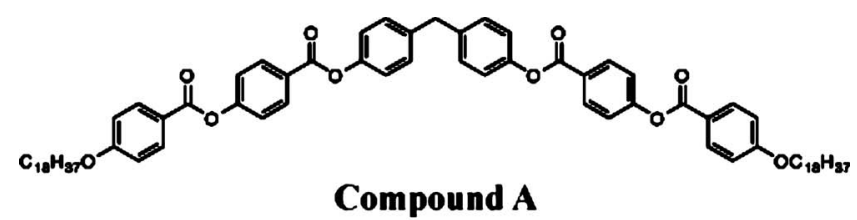

Iso $-161^{\circ} \mathrm{C}-\mathrm{Col}-147^{\circ} \mathrm{C}-\mathrm{SmCP}-114^{\circ} \mathrm{C}-$ Crystal

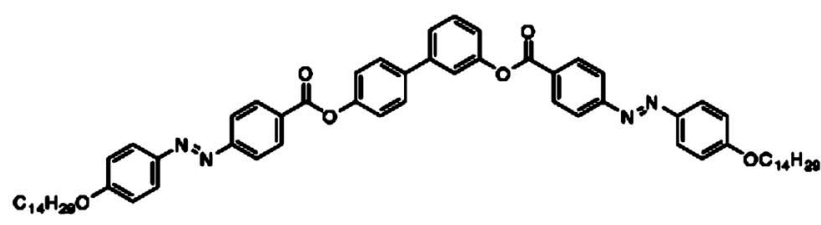

Compound B

Iso $-160^{\circ} \mathrm{C}-\mathrm{Col}-128^{\circ} \mathrm{C}-$ Crystal

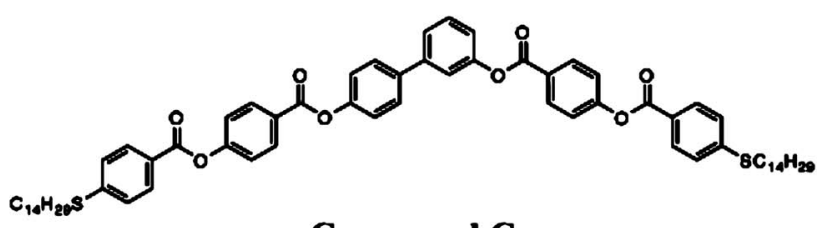

Compound C

Iso $-157^{\circ} \mathrm{C}-\mathrm{Col}-120^{\circ} \mathrm{C}-\mathrm{SmCP}-83^{\circ} \mathrm{C}-\mathrm{Crystal}$

FIG. 2. Molecular structures and phase sequence on heating [from differential scanning calorimetry (DSC) measurements] of compounds $\mathrm{A}, \mathrm{B}$, and $\mathrm{C}$ studied in this work. In compound $\mathrm{B}$ the transition from columnar to smectic could not be detected from DSC data as was in x-ray diffraction experiments. This effect could be attributed to the experimental conditions and the low transition enthalpy.

tion symmetry axis of the droplet about the vertical axis. Given the $\mathbf{a}^{*}$ and $\mathbf{c}^{*}$ vectors shown in the figure, gray dots correspond to reflections coming from the other domain and should not be indexed. Note that a pair of vertical and horizontal $\mathbf{a}^{*}, \mathbf{c}^{*}$, axes is not valid for indexing the observed black dots because, for example, the vertical coordinates of the (11), (02), or (13) peaks are not commensurate with those of the $(h h)$ reflections.

Compound B presents a very similar behavior to that of compound A, and the X-ray results are shown in Figs. 5(a) and 5(b). The parameters of the rectangular centered cell of the columnar phase are $a=70.7 \AA$ and $c=53.7 \AA$ (at $158{ }^{\circ} \mathrm{C}$ ) and the layer spacing in the smectic phase is $45.1 \AA$. The alignment is also kept during the transition as can be seen in Figs. 5(c) and 5(d). The only difference found was a small sudden change in the position of the (01) smectic peak with respect to the (11) columnar peak $[\Delta d=2.3 \AA$; see Fig. 5(a)]. In Ref. [6] a different indexation scheme was found for this material, presumably due to the interpretation of a B1-B2 coexistence as a single oblique B1 phase. Here, the inadequacy of horizontal and vertical $\mathbf{a}^{*}, \mathbf{c}^{*}$ axes is more 


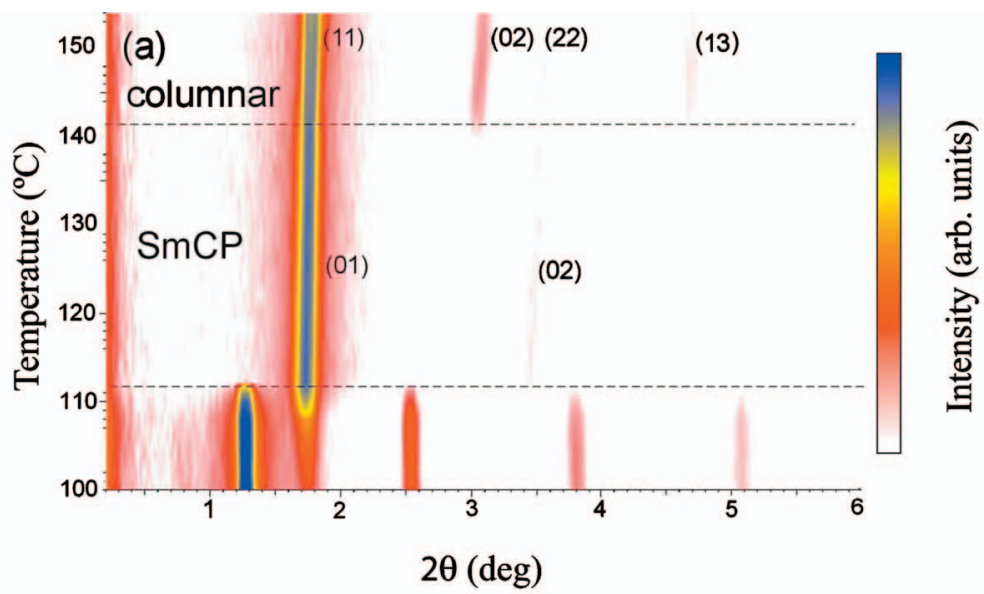

(b)
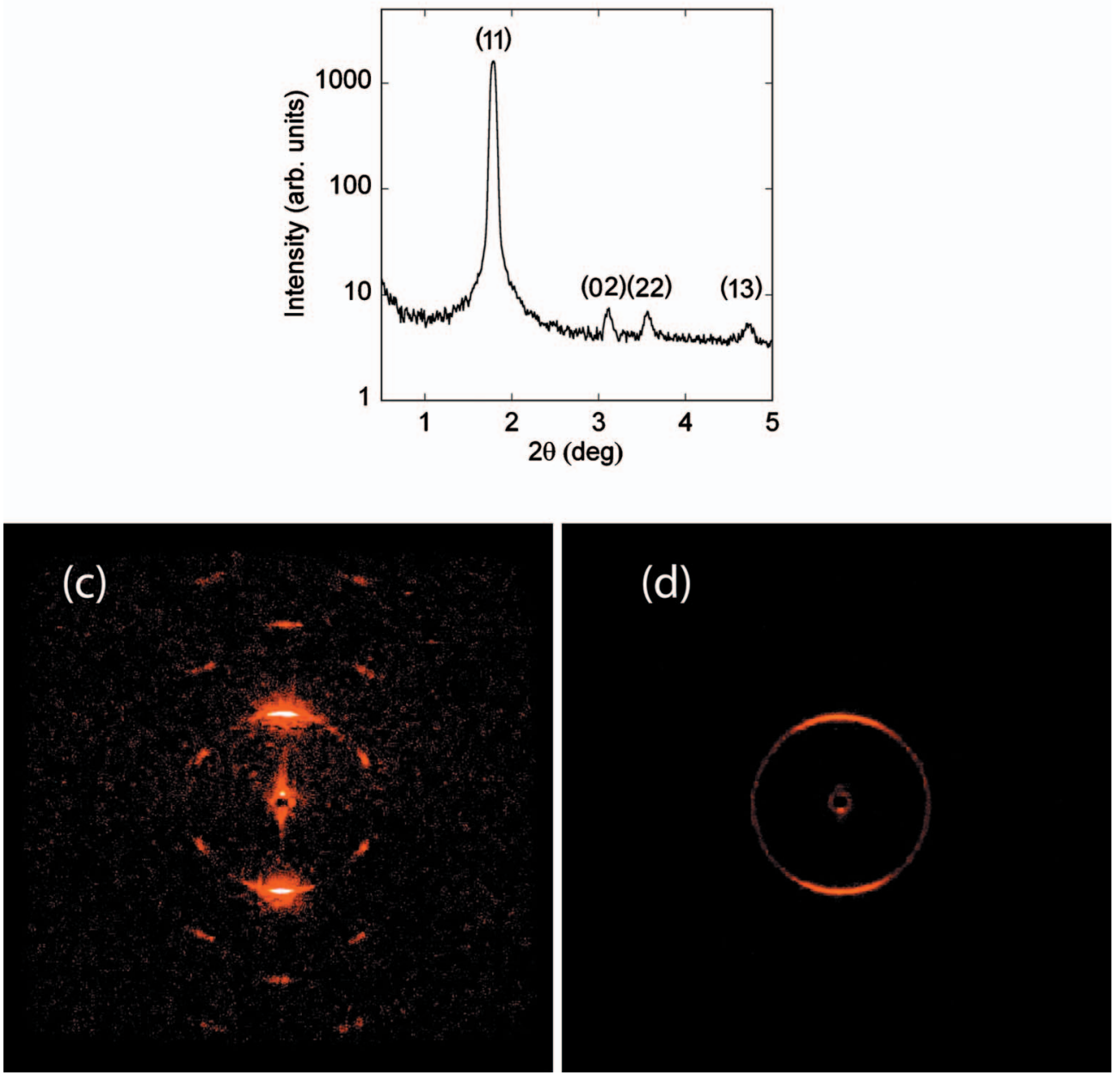

FIG. 3. (Color) (a) Azimuthally integrated 2D x-ray diffraction diagram vs temperature for compound A and its peak indexation. (b) X-ray intensity vs scattering angle at $149{ }^{\circ} \mathrm{C}$ in the B1 phase. (c) and (d) show two descriptive $2 \mathrm{D}$ x-ray diffraction diagrams of the columnar and smectic phases of compound $\mathrm{A}$, respectively.

evident than in the preceding case [see Figs. 4 and 5(c)].

Figure 6(a) shows the peak angular positions vs temperature of compound C. Figure 6(b) shows the powder diagram at $107{ }^{\circ} \mathrm{C}$ within the columnar phase. Positions of columnar (11) and smectic (01) peaks again coincide [as well as (22) and $(02)]$, but the variation of other peak positions in the columnar phase is noteworthy. In Fig. 7(a) the changes with temperature of the centered cell parameters $a, c$, and $\beta$ are shown. $c$ is constant through the columnar phase temperature range; but $a$, as well as $\beta$, undergoes important changes 


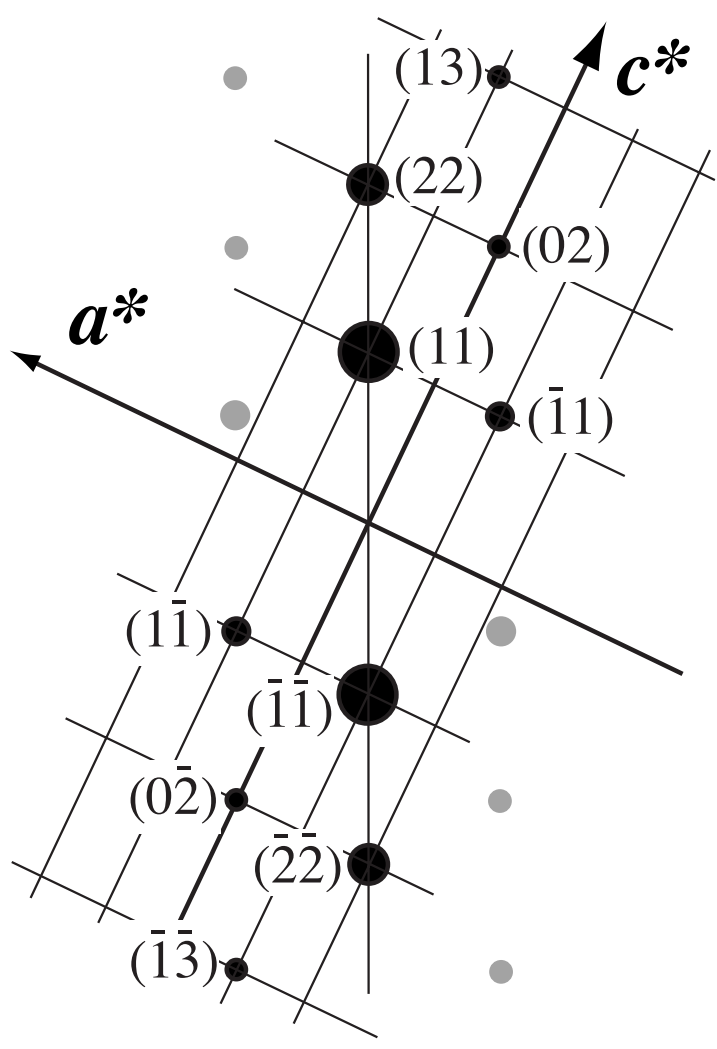

FIG. 4. Schematic representation of the x-ray diffraction diagram of compounds $\mathrm{A}$ and $\mathrm{B}$ showing the indexation for a $2 \mathrm{D}$ rectangular reciprocal lattice corresponding to one of the domains involved in the observed pattern. Gray dots are reflections that appear as a consequence of the revolution axis perpendicular to the drop surface and are originated in a different domain. These peaks can be indexed using a different pair of $\left(\mathbf{a}^{*}, \mathbf{c}^{*}\right)$ vectors related to those of the figure by a vertical twofold axis.

(from 70 to $134 \AA$ and from $85^{\circ}$ to $104^{\circ}$, respectively). The present indexation scheme contrasts with other previously reported for the same compound [14]. In that work the x-ray results were indexed on the basis of a centered rectangular cell. The disagreement can be mainly explained as due to the smaller angular resolution of that experiment, which prevented them from resolving peaks (11) and (111). Figures 6(c) and 6(d) show the 2D patterns at the B1 and Sm-CP phases. Again, the peaks in the B1 phase can be indexed as sketched in Fig. 4, although now the angle between $\mathbf{a}^{*}$ and $\mathbf{c}^{*}$ is not $90^{\circ}$ and depends on temperature [for the temperature shown in Fig. 6(c), $\mathbf{a}^{*}$ and $\mathbf{c}^{*}$ are nearly orthogonal, so the pattern is again similar to that of Fig. 4]. As in the above cases the alignment is kept at the transition. Figure $7(\mathrm{c})$ shows two illustrative electron density diagrams of the columnar phase of $\mathrm{C}$ at $143{ }^{\circ} \mathrm{C}$ and $103{ }^{\circ} \mathrm{C}$. They were constructed using the measured intensities following the procedure explained in [6]. Again, as is evident from Fig. 7(c), the smectic layers originate from the $(h h)$ planes as the parameters $a$ and $\beta$ increase.

\section{DISCUSSION}

As pointed out before, compounds $\mathrm{A}$ and $\mathrm{B}$ present a similar behavior, and although compound A will be used in

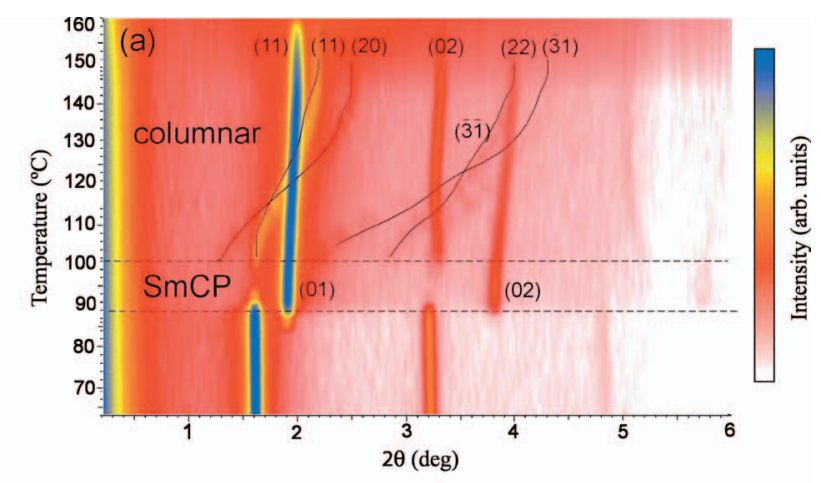

(b)
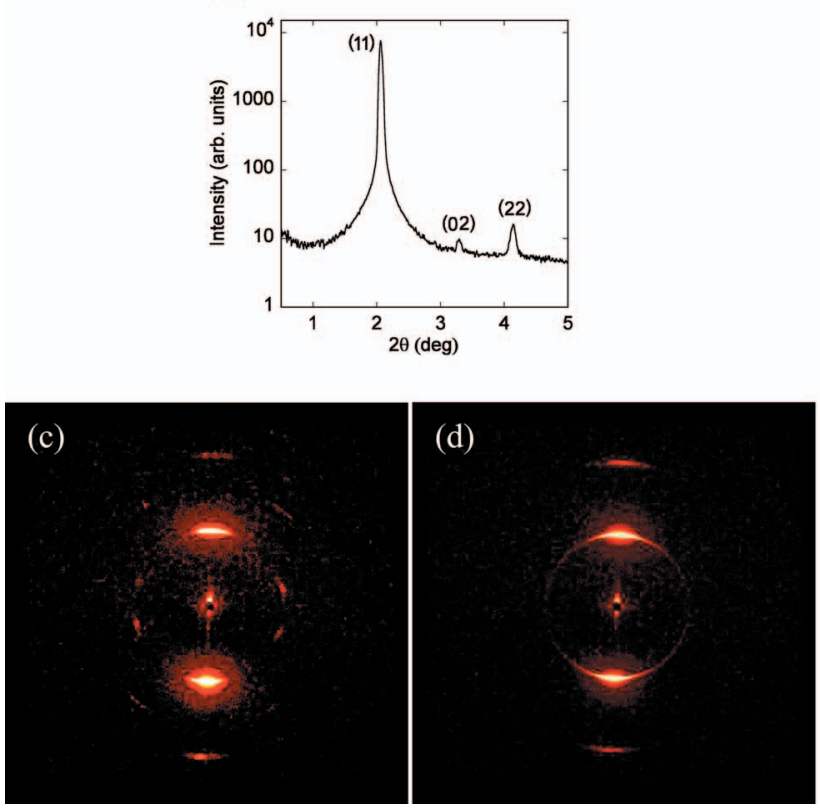

FIG. 5. (Color) (a) Azimuthally integrated 2D x-ray diffraction diagram vs temperature for compound $\mathrm{B}$ and its peak indexation. (b) X-ray intensity vs scattering angle at $157{ }^{\circ} \mathrm{C}$ in the $\mathrm{B} 1$ phase. (c) and (d) show two descriptive 2D x-ray diffraction diagrams of the columnar and smectic phases of compound B, respectively.

the explanation, the following results can be extended to compound B. According to the results of Figs. 3(c) and 3(d), the smectic layers of the $\mathrm{Sm}-\mathrm{CP}$ phase are parallel to the substrate and, therefore, this is also the case for the $(h h)$ crystallographic planes of the columnar phase. Since smectic layers will be formed in the Sm-CP phase from the $(h h)$ pseudolayers of the columnar phase, it appears reasonable to propose that blocks in these pseudolayers (at the same height in the drop) should have the same polarization direction. An ideal situation is depicted in Fig. 8 for compound A. In the figure the color of the block represents the two possibilities of the polarization direction. Rectangular blocks are tilted an angle $\alpha=\tan ^{-1}(c / a) \approx 30^{\circ}$ with respect to the normal of the substrate. As the centered cell is rectangular, molecules inside the blocks are parallel to the $c$ direction, giving rise to a perfect rectangular $\mathrm{Col}_{\mathrm{r}}$ phase. Figure 9(a) shows the generalization for an oblique $\mathrm{Col}_{\mathrm{ob}}$ phase. Blocks in adjoining corners of the centered cell have opposite polarization directions 


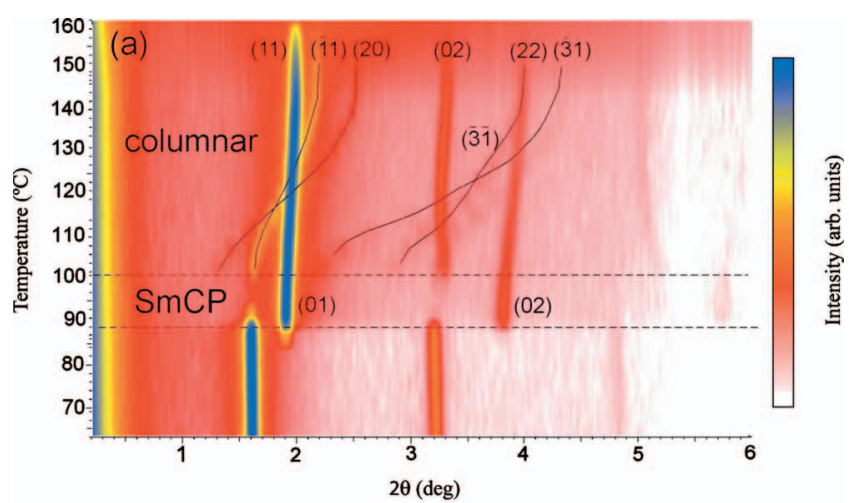

(b)
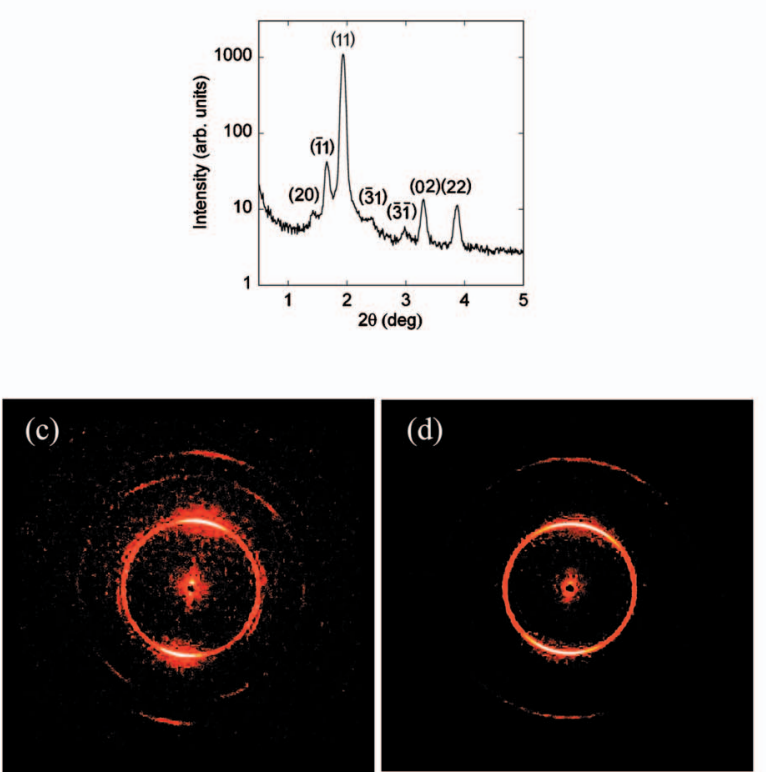

FIG. 6. (Color) (a) Azimuthally integrated 2D x-ray diffraction diagram vs temperature for compound $\mathrm{C}$ and its peak indexation. Black lines for some weaker peaks were included to show them clearly. (b) X-ray intensity vs scattering angle at $107^{\circ} \mathrm{C}$ in the $\mathrm{B} 1$ phase. (c) and (d) show two descriptive $2 \mathrm{D}$ x-ray diffraction diagrams of the columnar and smectic phases of a partially aligned sample of compound $\mathrm{C}$. The tilt of the direction where the most intense reflections appear in (b) and (c) with respect to the vertical is simply due to an inclination with respect to the horizontal plane of the substrate where the material drops were deposited.

(different colors in Fig. 8), contrary to the way the $\mathrm{Col}_{\mathrm{ob}}$ phase is usually thought of [Fig. 9(b)].

In view of the proposed molecular arrangement, the simplest and more logical transition mechanism could then be as follows. As the temperature is lowered, molecules inside each block slide up or down (see arrows in Fig. 10) maintaining the parallelism and making the "layer shift" between adjacent blocks smaller than half the length of a molecule. The blocks would not be rectangles anymore, but parallelograms (see Fig. 10). When the layer shift disappears, the blocks degenerate in perfect layers and, therefore, the $\mathrm{Sm}-C P$ phase is formed. It is evident that in the process the molecular tilt with respect to the layer remains almost constant. This transition mechanism gives rise to the right layer (a)

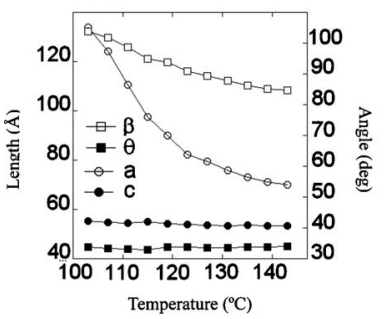

(c)

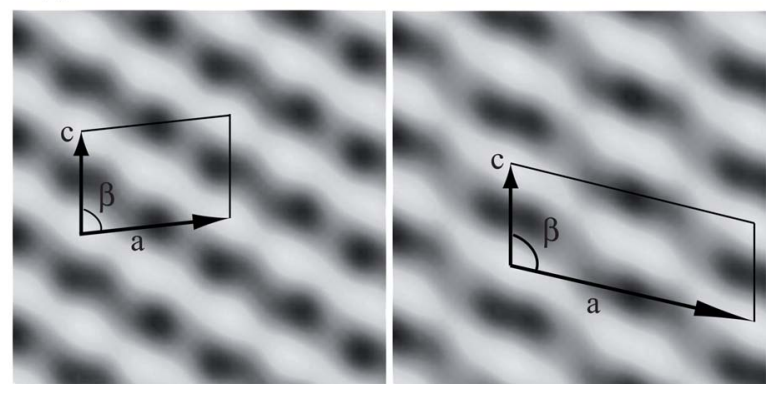

FIG. 7. (Color online) (a) Variations of the cell parameters $a, c$, and $\beta$ vs temperature for compound $\mathrm{C}$. Also the temperature dependence of the angle $\theta$ depicted in (b) is graphed. (c) Electron density of the columnar phase at $143{ }^{\circ} \mathrm{C}$ (left) and near the transition to the $\mathrm{Sm}-\mathrm{CP}$ phase $\left(103{ }^{\circ} \mathrm{C}\right)$ (right). The white regions represent areas with high electron density.

spacing ( $c \cos \alpha=49.2 \AA)$ in the Sm- $C P$ phase and would also explain the low enthalpy of the process. The structural change can even take place in a continuous fashion, meaning that the transition could be of second order.

The transition is clearly first order in compound B since there is a jump in the (11) spacing. In this case the rectangular blocks of the B1 phase are tilted by $\alpha=37^{\circ}$ with respect to the substrate normal. The opening angle of the blocks at the transition would be $90^{\circ}-\alpha=53^{\circ}$.

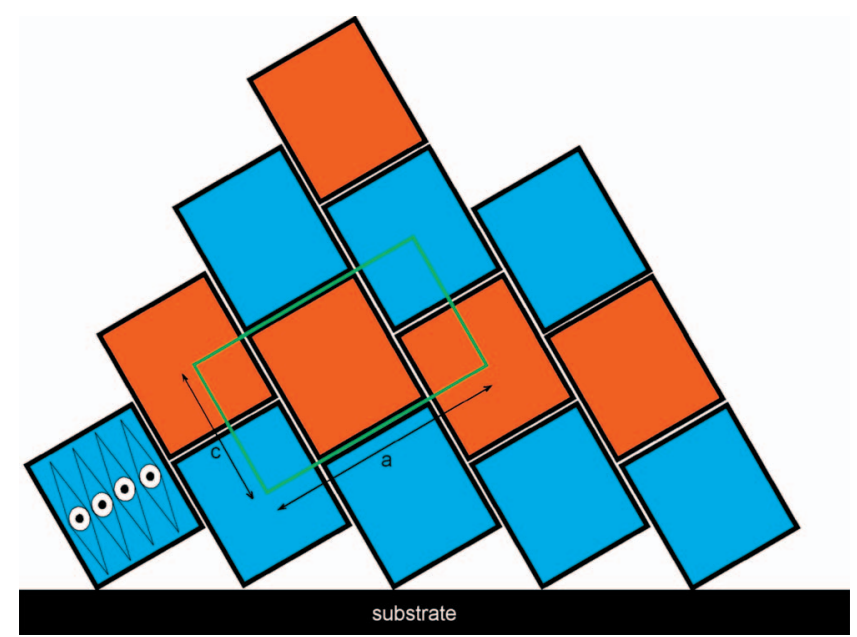

FIG. 8. (Color) Ideal arrangement of the blocks in an aligned drop of compound A. The molecules are orientated along the $c$ direction and the blocks are tilted an angle $\tan ^{-1}(c / a)$ with respect to the substrate normal. The color of the blocks represents the polarization directions, alternating in blocks at different drop heights. 
(a)

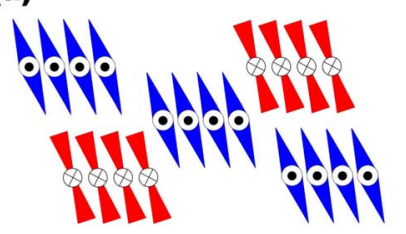

(b)

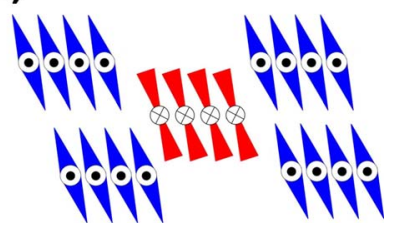

FIG. 9. (Color online) (a) Proposed structure for the $\mathrm{Col}_{\mathrm{ob}}$ phase of the compounds studied in this work and (b) typical $\mathrm{Col}_{\mathrm{ob}}$ $\left(\mathrm{B} 1_{\text {revtilt }}\right)$ structure found in the literature (see, for example, $[2,3]$ ).

In the case of compound $\mathrm{C}$ the process is somewhat different. Figure 7(b) shows the block structure compatible with the obtained x-ray results. In the figure the centered cell and the pseudolayer are also depicted. Two important points make a difference with respect to compounds A and B: on one hand, the strong dependence with temperature of $a$ and $\beta$ parameters and, on the other hand, the fact that the $\theta$ angle remains almost constant with temperature. The latter point can be checked by geometric considerations using the cell parameters of Fig. 7(a). This fact points toward a lamellarization process in which the blocks in the (11) pseudolayers of the columnar phase progressively stretch, so that $a$ and $\beta$ increase, keeping $c$ and $\theta$ constant. At the transition temperature the layer is perfectly formed. The calculated layer spacing in the smectic phase was $c \cos \theta=46 \AA$, which compares perfectly with the experimental one (46 $\AA$ ). As can be seen, the process leading to the smectic phase formation is different in this last compound to that of compounds $\mathrm{A}$ and $\mathrm{B}$; however, the proposed structure is still not the one in Fig. 9(b) but the one of Fig. 9(a) instead.

Summarizing the results for the three compounds, the classical structures both for the rectangular (compounds A and B) and oblique (compound C) columnar B1 phases have been discarded and, in their place, a different arrangement for the polarization blocks is proposed (see Fig. 9 for the oblique case). The actual symmetry group is monoclinic $p 2 / n$ (Fig. 11) even in the rectangular structures. If nonresonant $\mathrm{x}$-ray diffraction is used, all the blocks become

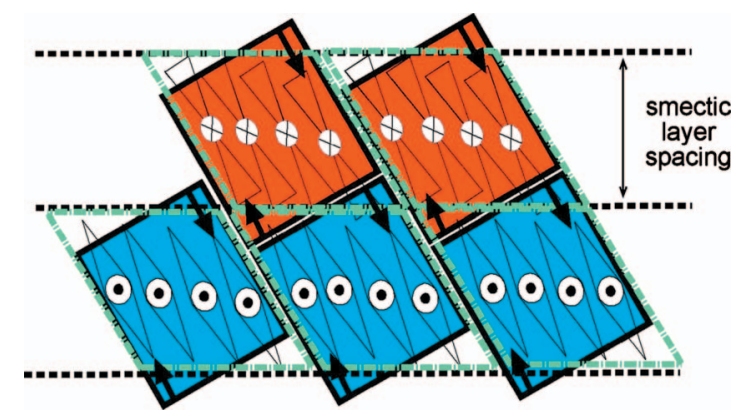

FIG. 10. (Color) Sketch of the change in the block shape through the transition from columnar to smectic phase. The solidlined rectangle represents the initial columnar state, and the greendashed parallelogram represents the final one. Black dashed lines depict the layers in the Sm-CP phase and the "pseudolayers" in the columnar one. Movement of the molecules in the blocks to form a smectic layer is depicted with arrows.
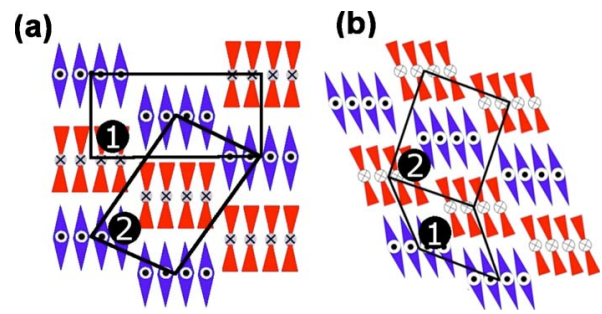

FIG. 11. (Color online) Different cell choices in the (a) rectangular and (b) oblique columnar B1 structures proposed. Cells (1) and (2) are appropriate for nonresonant and resonant x-ray diffractions, respectively.

indistinguishable and the apparent symmetry is monoclinic $\mathrm{p} 2 / \mathrm{m}\left(\mathrm{Col}_{\mathrm{ob}}\right)$ or orthorhombic $\mathrm{cmmm}\left(\mathrm{Col}_{\mathrm{r}}\right)$. Moreover, according to the proposed transition mechanism, the lowtemperature B2 phase should be racemic antiferroelectric, at least if the transition is continuous, i.e., the $\mathrm{Sm}-\mathrm{CP}$ variant should be $\mathrm{Sm}-C_{\mathrm{S}} P_{\mathrm{A}}$. Although some reports have appeared pointing toward a homochiral Sm- $C_{\mathrm{A}} P_{\mathrm{A}}$ phase in compounds $B$ [6] and C [9], it is important to remark that these experiments have always been carried out under electric field. Indeed the character of the $\mathrm{Sm}-\mathrm{CP}$ phase ground state is hard to be determined by texture observation, and only after electric field application well-developed domains appear. On the other hand, the electric field is likely to provoke deracemization of the structure [17], which would give rise to a $\mathrm{Sm}-C_{\mathrm{A}} P_{\mathrm{A}}$ phase. The nature of the phase in the absence of field is currently under investigation using resonant $\mathrm{x}$-ray diffraction. Preliminary results indicate that the B2 phase is actually $\mathrm{Sm}-C_{\mathrm{S}} P_{\mathrm{A}}$. A deeper analysis of this point together with the examination of the $\mathrm{B} 1$ phase under the resonant $\mathrm{x}$-ray technique is our objective for the near future.

The field-induced B1-B2 transition takes place through a different mechanism. Under an electric field, the resulting B2 phase must be ferroelectric, most probably $\mathrm{Sm}-C_{\mathrm{S}} P_{\mathrm{F}}$, and requires the polarization flipping of half the columns. This flipping does not occur when the transition is thermally driven and has no counterpart in our model.

\section{CONCLUSIONS}

In conclusion, three bent-core materials possessing a columnar to $\mathrm{Sm}-C P$ phase transition have been studied by $\mathrm{x}$-ray diffraction in aligned samples. The temperature dependence of the diffraction patterns has led us to propose a mechanism for this transition, which accounts for the low enthalpy usually observed in this process. In addition, the relative orientation of the dipoles among the blocks of the columnar phases has been reexamined proving that these structures show a large degree of lamellarization with pseudosmectic ferroelectric layers along the (11) crystallographic planes.

\section{ACKNOWLEDGMENTS}

Measurements in this work were carried out using the facilities in the liquid crystal group of Ewa Gorecka (Warsaw 
University). This research was supported by CICYT-FEDER of Spain-UE Contracts No. MAT2008-06522-CO2 and No. MAT2009-14636-CO3, the Aragón Government (E04), and the Basque Country Government (Project No. GIC10/45). N.G. acknowledges the Spanish Government for support via the Juan de la Cierva program.
[1] G. Pelzl, S. Diele, and W. Weissflog, Adv. Mater. 11, 707 (1999).

[2] R. Amaranatha Reddy and C. Tschierske, J. Mater. Chem. 16, 907 (2006).

[3] H. Takezoe and Y. Takanishi, Jpn. J. Appl. Phys., Part 1 45, 597 (2006).

[4] D. A. Coleman et al., Science 301, 1204 (2003).

[5] N. Vaupotic and M. Copic, Phys. Rev. E 72, 031701 (2005).

[6] C. L. Folcia et al., Chem. Mater. 18, 4617 (2006).

[7] E. Gorecka, N. Vaupotic, and D. Pociecha, Chem. Mater. 19, 3027 (2007).

[8] H. F. Gleeson and L. S. Hirst, ChemPhysChem 7, 321 (2006).

[9] J. Ortega, M. R. de la Fuente, J. Etxebarria, C. L. Folcia, S.
Diez, J. A. Gallastegui, N. Gimeno, M. B. Ros, and M. A. Perez-Jubindo, Phys. Rev. E 69, 011703 (2004).

[10] G. Dantlgraber et al., Chem. Mater. 14, 1149 (2002).

[11] H. N. Shreenivasa Murthy et al., Liq. Cryst. 31, 1347 (2004).

[12] H. N. Shreenivasa Murthy et al., J. Mater. Chem. 16, 1634 (2006).

[13] J. Kirchhoff et al., Appl. Phys. Lett. 90, 161905 (2007).

[14] C. L. Folcia, J. Etxebarria, J. Ortega, and M. B. Ros, Phys. Rev. E 74, 031702 (2006).

[15] N. Gimeno et al., Chem. Mater. 21, 4620 (2009).

[16] I. C. Pintre et al., J. Mater. Chem. 17, 2219 (2007).

[17] R. Pyc et al., J. Mater. Chem. 14, 2374 (2004). 